\title{
Anatomically Informed Convolution Kernels for the Projection of fMRI Data on the Cortical Surface
}

\author{
Grégory Operto ${ }^{1}$, Rémy Bulot ${ }^{1}$, Jean-Luc Anton ${ }^{2}$, and Olivier Coulon ${ }^{1}$ \\ ${ }^{1}$ Laboratoire LSIS, UMR 6168, CNRS, Marseille, France \\ ${ }^{2}$ Centre IRMf de Marseille, Marseille, France
}

\begin{abstract}
We present here a method that aims at producing representations of functional brain data on the cortical surface from functional MRI volumes. Such representations are required for subsequent corticalbased functional analysis. We propose a projection technique based on the definition, around each node of the grey/white matter interface mesh, of convolution kernels whose shape and distribution rely on the geometry of the local anatomy. For one anatomy, a set of convolution kernels is computed that can be used to project any functional data registered with this anatomy. The method is presented together with experiments on synthetic data and real statistical t-maps.
\end{abstract}

\section{Introduction}

The cerebral cortex is known to generate most of the activity measured by functional techniques, such as functional magnetic resonance imaging (fMRI). Despite its sheet-like nearly two-dimensional structure, methods for functional data analysis still widely consider the cortex in its original three-dimensional grid. The surface-based approach is though an attractive approach for intersubject matching 35] or statistical analysis 4962], coping with its highly folded nature. However, a problem which has remained quite ignored by the litterature concerns the projection of functional values onto the cortical surface, although a relevant description of the volume-based signal on a two-dimensional model is essential to a subsequent cortical-based analysis. Some methods propose to interpolate intensities along a normal direction or inside a sphere centered at each node of the mesh representing cortical surface (e.g. when using free package Brainvisa [1]), or to assign each node with the value of its containing voxel [13], or to compute trilinear interpolations 22. On the other hand, other methods try to embed some explicit anatomical information into the process. For example, [14] defines each node's influence scope through voxels distances to and along the cortical mesh. In [7, a geodesic Voronoï diagram is computed so that each node value is integrated within an associated set of voxels defined by the local anatomical geometry.

From these methods stems a common interest in delineating 3D areas, sometimes overlapping, onto which the signal emanating from the nodes would possibly be dispersed, taking local anatomical features into account. This 
consideration was a starting point for developing a new method, based on an expected, anatomically related, signal distribution in BOLD-EPI volumes, leading to surface-based representations of the cortical ribbon activity. This paper details in section 2 the model which the method relies on. Section 3 presents the method itself. Results and discussion are then detailed in section 4 .

\section{Method}

\subsection{Model of the Expected Signal Distribution}

We consider two distinct phenomena in order to assess the expected distribution of BOLD signal around a single node of the cortical mesh, with physiological and image- related motivations. First, as a consequence of the columnar architecture of neurons inside the cortex [12, we expect each cortical column to show homogeneous intrinsic activity, suggesting that this activity and the signal should be constant in the column orientation, i.e. normal to the surface. Then, since neighborhood connections between columns lead to strong interactions along the surface, induced decaying activity around a functional activation focus can be measured [8]. Beside this, the image acquisition process inevitably induces the signal to sparsely spill outside the cortex, possibly mixing-up sources and bringing signal to anomalous areas. These observations resultantly inspired our method as it aims to figure out in which proportions neighbouring voxels can be associated with the expected signal at a surface node and hence in which proportions their intensities should weigh on the interpolated node value. In respect with our assumptions, the distribution of activity on the cortical surface is thus depicted as a function of distance parameters along two main directions, normal and parallel (geodesic distance) to the surface, as shown on figure 1.

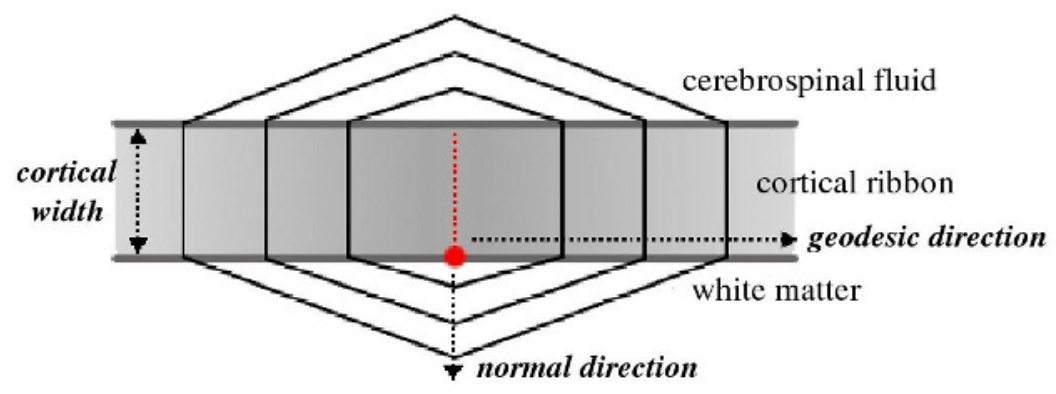

Fig. 1. Expected distribution of activity around a given point of the cortical surface presented with iso-influence curves

\subsection{Computing the Convolution Kernels}

After extracting the white/gray matter interface triangulation from MR T1 anatomical image using the Brainvisa package [10] and operating registration between functional volumes and anatomy, the first processing step deals with 
the evaluation, around each node $n_{c}$, of influence factors for any surrounding voxel. The process is done on isotropic voxels so as to cope with the anisotropy of the MRI spatial point spread function. Since influences are here related to distances, we compute two distances, geodesic and normal, between the node $n_{c}$ and each voxel $v_{i}$ of a neighborhood $V_{c}$ around $n_{c}$. These two distances are then used as inputs of two distinct weight functions, both of them describing a linear influence decay as distance increases. This results in associating a specific convolution mask to each node. Computing $15 \times 15 \times 15$ voxels mask at anatomical resolution $(1 \times 1 \times 1 \mathrm{~mm})$, the covered area is wide enough so any outer voxel has a null weight.

The computation of normal distances relies on the propagation of a proximity information, starting from the voxel $v_{c}$ containing the current node $n_{c}$. To perform this, we use a Fast Marching-like distance propagation algorithm adapted from the method described in [11]. The idea is to propagate a front that attributes to each surrounding voxel $v$ its normal distance to the surface $d_{v}$, and its closest node on the surface $n_{v}$ (with $d_{v}$ being approximated to the Euclidean distance between $v$ and $n_{v}$ ). The voxel $v_{\text {current }}$ of the front which is the closest to the surface is considered processed and its neighbors $v_{i}$ are added to the front, except those already processed. For each $v_{i}$, it is assumed that the associated $n_{v_{i}}$ is in a close neighborhood of $n_{v_{\text {current }}}$. Once $n_{v_{i}}$ is found, $d_{v_{i}}$ is computed and both are associated with $v_{i}$. The other neighbors of $v_{\text {current }}$ are processed in a similar manner. Once all neighbors processed, the algorithm is iterated with the voxel having the lowest $d_{v}$. The algorithm is initialized with the nearest voxel $v_{c}$ to the considered node $n_{c}$. This process is illustrated on figure 2.
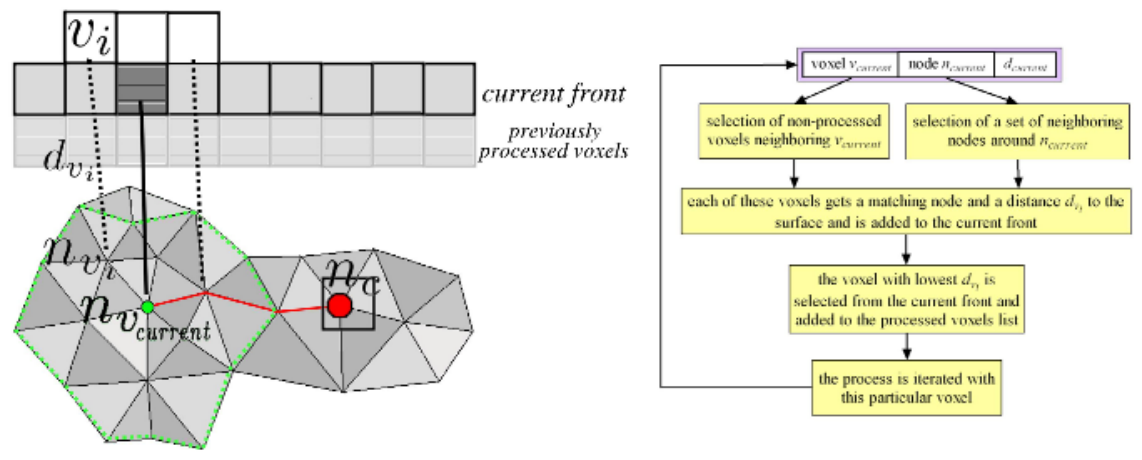

Fig. 2. (left) Computation of normal (black lines) and geodesic (red line) distances using a Fast Marching-like algorithm 11] - (right) Flowchart of the algorithm

Once every surrounding voxel was assigned its proximity index to the surface, each of them gets an estimated geodesic distance between $n_{c}$ and the node $n_{v}$ from which the normal distance was computed at first. A shortest path algorithm creates from $n_{c}$ a geodesic distance map covering the whole area under the influence of its activity. 
The voxels characterized by two distances are then given weights by the combination of two functions described on figure 3 . Geodesically, we estimate that a node located beyond $6 \mathrm{~mm}$ far from $n_{c}$ is no longer under influence : aside from its physiological justification, this user-specified distance allows to handle the smoothness of the final projection, similar to the variance of the basis functions defined by 9 . On the other hand, the normal distance weight depends directly on the anatomical structure the voxel is located in. The normal weight of a voxel is maximal if located in the cortical ribbon and decreases outside to reach zero at a user-specified normal distance of $3 \mathrm{~mm}$ (figure 3). As a result, each voxel $v$ gets assigned a value $\omega(v)$ corresponding to the product of its two weights. Finally, each mask is normalized so that the sum of all weights equals 1 , for energy
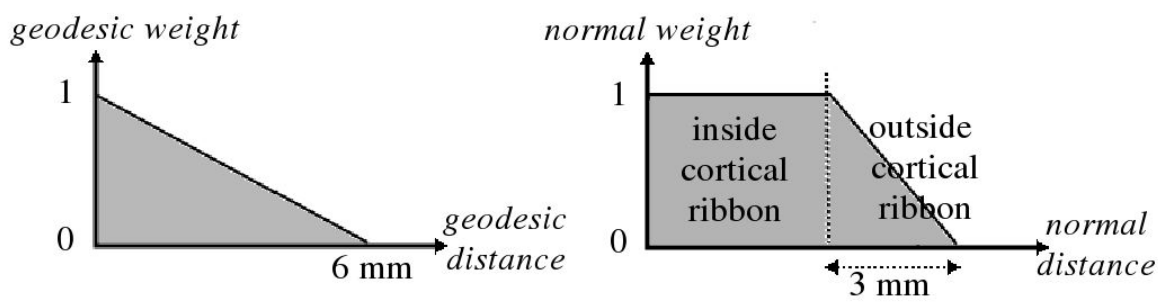

Fig. 3. Geodesic (left) and normal (right) weight functions

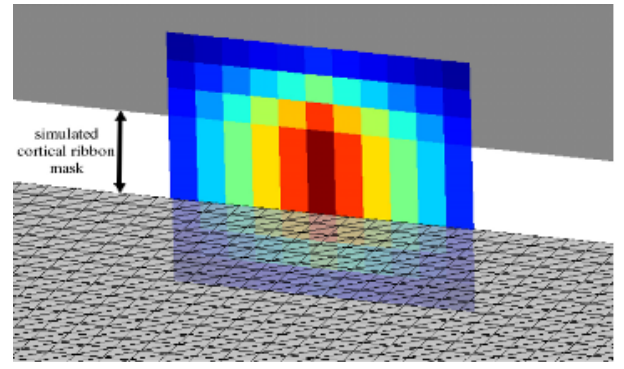

Fig. 4. Convolution kernels obtained for a node on a flat surface mesh with superimposed simulated cortical mask
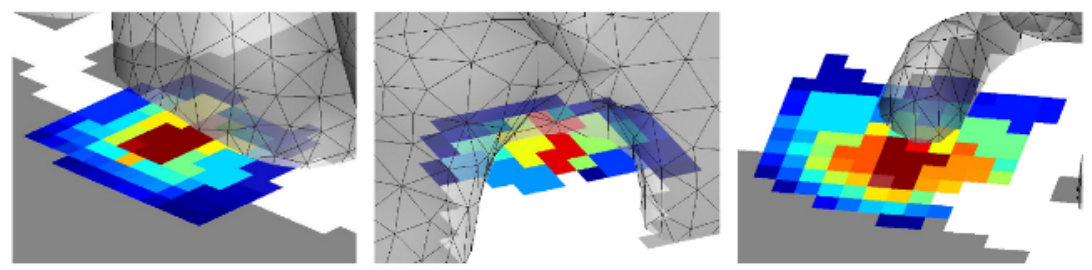

Fig. 5. Convolution kernels obtained for nodes on real cortical meshes with superimposed cortical masks 
and intensity range preservation purposes. Figure 4 displays a convolution kernel computed on a flat synthetic surface mesh, showing a plateau corresponding to the cortical ribbon. Figure 5 shows on real cortical meshes three kernels with shapes strongly influenced by local anatomy.

\subsection{Projecting the Functional Data}

Once the convolution kernels were computed for the whole mesh, we then integrate the voxel intensities onto the surface nodes : since any surrounding voxel has an estimated influence on the node, the value $s\left(n_{s}\right)$ assigned to the node $n_{s}$ is the result of a linear combination expressed as following :

$$
s\left(n_{s}\right)=\sum_{V_{i}} \omega_{s}\left(v_{i}\right) \cdot I\left(v_{i}\right)
$$

where $V_{i}$ is the voxels area within which influences on $n_{s}$ were evaluated, $\omega_{s}\left(v_{i}\right)$ is the computed weight for voxel $v_{i}$ and $I\left(v_{i}\right)$ is $v_{i}$ 's intensity on the functional image. The method is designed so that the costly step of computing the masks is done only once by subject, then any functional image can immediatly be projected onto the cortical surface.

\section{Results and Discussion}

The method is applicable either to raw functional data, opening the way to cortical surface-based statistical analysis, or to the results of a standard fMRI analysis, as for instance volumic t-maps of activation detection. We tested our algorithm on images from a somatotopy experiment in which 17 subjects observed 6 different sensorimotor tasks, involving foot, elbow, thumb, index, little finger and tongue. EPI scans were then analyzed using SPM'99 with task/rest contrasts. Figure 6 presents a projection of a SPM volume-based t-map from elbow activation onto its corresponding cortical mesh, then displayed on inflated mesh, then illustrates differences between our projection and the results of a classical method which interpolates values within spheres centered at each node.

In comparison to methods which interpolate values along normal directions, or within voxels intersecting the cortical ribbon [13], our projection takes account of more voxels in respect with local anatomy and their intensities influences are differential within the interpolation area. Relating voxels to surface nodes using geodesic distances is a relevant way to deal with the folded nature of cortex, rather than euclidean distances. However, the propagation of distance information as we perform it, giving each voxel a matching node on the surface from which geodesic and normal distances are computed, implies a geometrical coherence between these nodes and therefore robustness to errors in registrating the functional volumes to anatomy. On the contrary, the use of a closest point criterium (through geodesic distances calculation as in 14, or Voronoï-diagrams definition as in [7) makes methods sensitive to these errors since very slight 

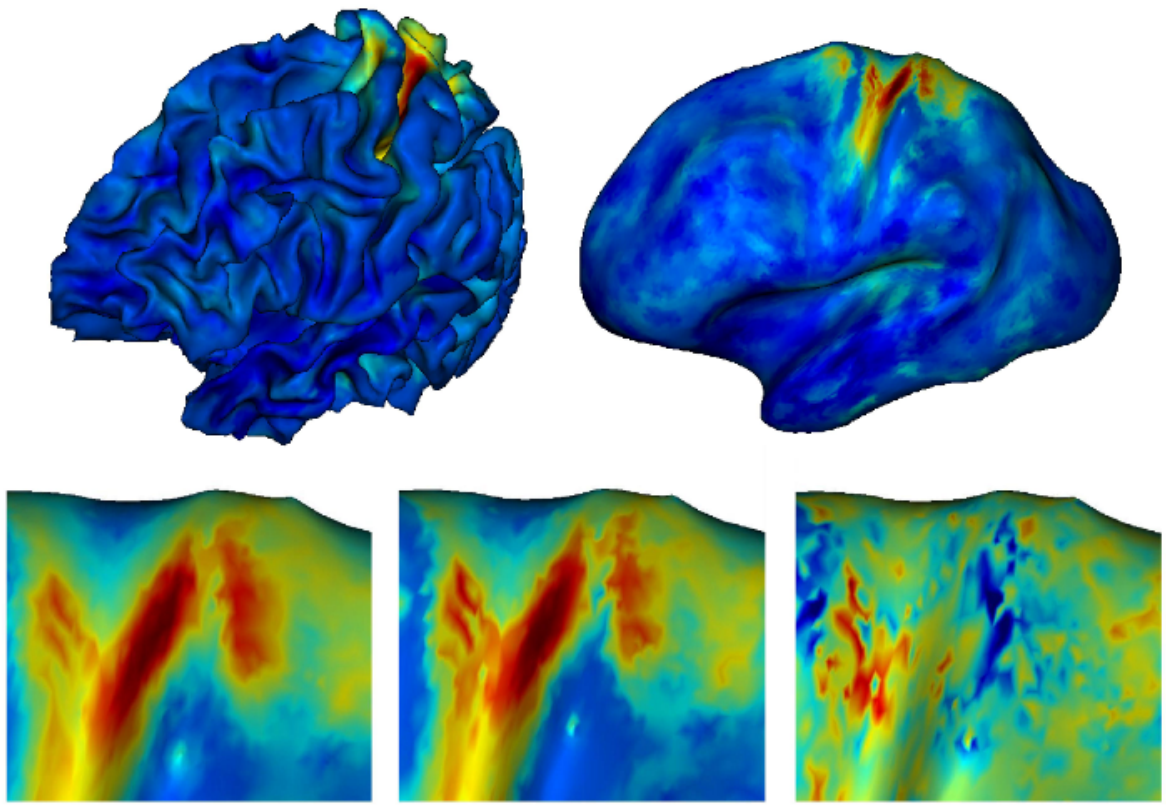

Fig. 6. Projection of a SPM t-map onto its matching cortical mesh, presented on (top) original and inflated surfaces. (bottom) Detailed view of a same area : (left) using sphere interpolation (middle) using convolution kernels (right) difference texture between the two methods results.

translations between functional volumes and anatomy will get some particular voxels totally different matching nodes. This is observed in certain regions e.g. near sulcal bottom lines where curvature varies much and where several nodes are quasi-equidistant to some voxels. In these cases, our method gives implicit preference to nodes on the same side of sulcus as the node at the center of the current kernel.

Assigning each voxel with weights results in overlapping convolution kernels from a node to another, which is different from parcelling the cortical ribbon into distinct cells. In our model, we consider a voxel's scope is not limited to one node even its closest one. On this point, our technique gathers the advantages from overlapping interpolation spheres and anatomically-informed Voronoï cells.

On figure 6. a volumic SPM t-map was projected using our algorithm, displaying relevant motor activations, similar to results with interpolation within spheres. The difference texture between the two projections shows that our method assigns smaller values in the region (colored in blue) between the two depicted activations which are actually located each on one side of a gyrus. This illustrates its ability to separate different geodesically distant activations and to avoid mixing-up signals from different sources. 


\section{Conclusion and Further Work}

In this paper, we propose a method allowing the projection of functional images onto the cortical surface. In application to raw data, the produced surface-based representations of the BOLD images are essential in the framework of corticalrestricted statistical analysis methodologies, such as Cortical Surface Mapping 2. Applying it to activation maps can serve visualisation purposes, as well as cortical localisation of activation foci through the use of a surface-based coordinate system [53, in comparison to localisation in 3D normalized spaces.

This work will be extended to validation stages, e.g. evaluating the method robustness to functional/anatomical registration errors or its dependence on the mesh spatial resolution and on nodes positions, after which it will take part in future cortical-based functional localisation experiments.

\section{References}

1. http://brainvisa.info.

2. A. Andrade, F. Kherif, J.-F. Mangin, K. Worsley, A.-L. Paradis, O. Simon, S. Dehaene, and J.-B. Poline. Detection of fMRI activation using cortical surface mapping. Hum. Brain Mapp., 12:79-93, 2001.

3. C. Clouchoux, O. Coulon, D. Riviere, A. Cachia, J.-F. Mangin, and J. Regis. Anatomically constrained surface parameterization for cortical localization. In J. Duncan and G. Gerig, editors, LNCS - Medical Image Computing and ComputerAssisted Intervention - MICCAI 2005: 8th International Conference, volume 3750, pages 344-351. Springer-Verlag Berlin Heidelberg, october 2005.

4. T.G.M. Van Erp, V.Y. Rao, H.L. Tran, K.M. Hayashi, T.D. Cannon, A.W. Toga, and P.M. Thompson. Surface-based analysis of functional magnetic resonance imaging data. In J. Duncan and G. Gerig, editors, LNCS - Medical Image Computing and Computer-Assisted Intervention - MICCAI 2004: 7th International Conference. Springer-Verlag Berlin Heidelberg, 2004.

5. B. Fischl, M.I. Sereno, R. Tootell, and A.M. Dale. Cortical surface-based analysis, ii: Inflation, flattening, and a surface-based coordinate system. NeuroImage, 9:195207, 1999.

6. R. Goebel and W. Singer. Cortical surface-based statistical analysis of functional magnetic resonance imaging data. Neuroimage, 9:S64, 1999.

7. C. Grova, S. Makni, G. Flandin, P. Ciuciu, J. Gotman, and J.-B. Poline. Anatomically informed interpolation of fMRI data on the cortical surface. Neuroimage, in press, 2005.

8. P.B. Johnson, S. Ferraina, and R. Caminiti. Cortical networks for visual reaching. In Experimental Brain Research, volume 97, pages 361-365, 1993.

9. S.J. Kiebel, R. Goebel, and K.J. Friston. Anatomically informed basis functions. NeuroImage, 11(6.1):656-667, 2000.

10. J.-F. Mangin, V. Frouin, I. Bloch, J. Régis, and J. López-Krahe. From 3D magnetic resonance images to structural representations of the cortex topography using topology preserving deformations. Journal of Mathematical Imaging and Vision, 5:297-318, 1995.

11. S. Mauch and D. Breen. A fast algorithm for computing the closest point and distance function. Technical report unpublished, CalTech, 2000. 
12. V.B Mountcastle. An organizing principle for cerebral function: The unit module and the distributed system. The mindful brain: Cortical organization and the group selective theory of higher brain function, page 7, 1978.

13. Z. Saad, C. Reynolds, B. Argall, S. Japee, and R.W. Cox. Suma: an interface for surface-based intra- and inter-subject analysis with afni. In Proc. IEEE Intl. Symp. Biomedical Imaging, page 1510, 2004.

14. J. Warnking, M. Dojat, A. Gurin-Dugu, C. Delon-Martin, S. Olympieff, N. Richard, A. Chhikian, and C. Segebarth. fmri retinotopic mapping-step by step. Neuroimage, 17(4):1665-1683, December 2002. 